\title{
Evaluación del transporte público en el Estado de México. El caso del Mexibus $^{1}$
}

JATME LiNARES ZaRCo*

\section{RESUMEN}

El desarrollo del capitalismo no se explica sin la evolución del transporte, y viceversa. Sin embargo, la gobernanza en países subdesarrollados, como México, se enfrenta a una crisis económica y urbana permanente, que limita los alcances de las políticas públicas del transporte urbano. Durante la última década, el origen y evolución del sistema Mexibús, en el Estado de México, responde a la creciente necesidad de movilidad que diariamente enfrentan más de 10 millones de mexiquenses de los municipios conurbados de la Zona Metropolitana de la Ciudad de México (ZMCM). A casi una década de la puesta en operación de la primera línea del sistema de transporte público (Bus Rapid Transit, por sus siglas en inglés), resulta conveniente evaluar el impacto económico y social que ha tenido entre los habitantes del Valle de México.

Palabras clave: crisis económica, crisis urbana, saturación urbana, transporte público.

Clasificación JEL: R, R41.

Síntesis de la investigación: "El sistema del Transporte Público en el Estado de México. El caso de las líneas 1, 2 y 3 del Mexibús" Coordinada por el autor, publicada por la FES AragónUNAM, 2019.

* Facultad de Estudios Superiores Aragón, Universidad Nacional Autónoma de México, México. Correo electrónico: jlinares112@yahoo.com.mx. 


\section{ABStRACT}

\section{Evaluation of Public Transport in the State of Mexico. The Mexibus Case}

The development of capitalism cannot be explained without the evolution of transportation and vice versa. However, governance in underdeveloped countries such as Mexico faces a permanent economic and urban crisis, which limits the scope of public policies for urban transport. The origin and evolution of the mexibus urban transport system in the State of Mexico in the last decade responds to the growing need for urban mobility that more than 10 million people living in the urbanized municipalities of the Metropolitan Zone of Mexico City (ZMCM) face on a daily basis. Almost a decade after the start of the first line of the Rapid Transit Bus, it is convenient to evaluate the economic and social impact that this transport has had among the inhabitants of the Valley of Mexico.

Keywords: economic crisis, urban crisis, urban saturation, public transport.

JEL Classification: R, R41.

\section{INTRODUCCIÓN}

El estudio del transporte urbano ha estado presente en la agenda de los grandes cronistas de México, "desde el paseo a caballo escrita por Francisco Cervantes en 1554, Manuel Payno cronicó la ciudad de la diligencia en 1848, cuarenta años después M. Gutiérrez Nájera lo hizo en tranvía" (De Mauleón, 2018: 108);sin olvidar a Angel del Campo (micros) quien habló sobre la bicicleta hacia finales del siglo XIX. A mediados del XX, el cronista musical Chava Flores escribió la canción "Voy en el metro", en la que describe el servicio de los camiones versus la modernidad del metro.

En el Estado de México, el desarrollo del transporte público va desde finales del siglo XIX hasta comienzos del XX, con el uso de la tracción animal. Al concluir el movimiento armado de 1910-1917 y pasados los estragos de la crisis económica mundial de 1929-1933, surge el transporte público urbano impulsado por el motor de combustión interna, paralelamente al desarrollo del sector industrial, que se fue 
convirtiendo en el propulsor económico del país, generador de ingresos y de empleo, así como uno de los factores de atracción del creciente flujo migratorio campo-ciudad. "La migración a las ciudades registró su mayor volumen y ritmo durante el periodo de urbanización rápida 1940-1970, al totalizar 6.1 millones de personas, de las cuales, 1.6 millones se produjeron entre 1940 a 1950, 1.8 millones durante 19501960 y 2.7 millones de 1960 a 1970" (Unikel, 1974: 54).

Los crecientes flujos migratorios pronto se tradujeron en altos niveles de concentración de la población en algunas ciudades del país, entre ellas la Ciudad de México, situación que implicó un mayor desarrollo de los sistemas de transporte, incluyendo el ferrocarril. "De esta forma, la vocación industrial de las delegaciones de Azcapotzalco, Gustavo A. Madero, Hidalgo y otras más, se vio acrecentada gracias a la disponibilidad de suelo y sobre todo a las vías de ferrocarril existente" (Conapo, 1998: 27). La lógica de expansión de la mancha urbana pronto rebasó la frontera de la Ciudad de México para extenderse hacia Naucalpan y Tlalnepantla, en el Estado de México, por lo que en 1950 el servicio de taxis colectivos de ruta fija se une a los autobuses urbanos para atender la creciente demanda de movilidad cercana a los 60 mil habitantes de los dos municipios señalados.

En la década de los setenta, mientras estaba en curso lo que Garza llama la urbanización acelerada (1990: 6), la Zona Metropolitana de la Ciudad de México (ZMCM) se había expandido aún más hacia la periferia, hasta integrar 11 municipios conurbados, cuya suma ascendía a aproximadamente dos millones de habitantes, que demandaban un creciente número de unidades de transporte, tanto de taxis colectivos como de camiones urbanos; sin embargo, ante el aumento en el déficit del transporte y el incremento de la población, a finales de los setenta e inicios de los ochenta, se pone en marcha el sistema de transporte troncal del Estado de México, que permitiría complementar y alimentar Cuatro Caminos de la Línea 2, primera estación del STC metro localizada en el Estado de México, a la cual posteriormente se agregaron el servicio de combis y microbuses.

En la década de los noventa del siglo XX, ante el crecimiento desbordante de la mancha urbana de "la ZMCM, la cual comprendía ya 27 municipios conurbados del Estado de México y 16 delegaciones del D.F., conjuntamente sumaban más de 15 millones de habitantes" (Conapo, 1998: 27), el número de unidades de transporte público, 
formado por camiones, taxis colectivos, combis y microbuses, se había incrementado considerablemente, además de haberse puesto en operación de la línea "A" del Metro que llegaba al municipio de La Paz en 1991.

Al finalizar el siglo XX, cuando "la ZMCM concentraba 17.9 millones de habitantes 8.6 millones en el D.F. y 9.3 millones de distribuidos entre 41 municipios del Estado de México y uno de Hidalgo" (Linares, 2009: 139), el número de unidades de transporte público se había duplicado con el propósito de atender la demanda creciente de movilidad urbana; adicionalmente, el STC Metro seguía penetrando en territorio mexiquense mediante la edificación de ocho estaciones de la línea B que han permitido transportar habitantes entre Nezahualcóyotl y Ecatepec, principalmente.

Previo a la entrada en operación de la línea 1 del Mexibús, en la entidad se generaban 9.2 millones de viajes al día, de los cuales 4.3 millones se hacían en transporte público y 4.9 millones de personas se desplazaban en transporte privado. El primero se caracterizaba por la obsolescencia del parque vehicular, la sobreoferta con unidades de baja capacidad, así como la incompatibilidad entre sus diferentes modos de transporte, además de que las asociaciones de transportistas no estaban debidamente conformadas en empresas formales. Es decir, la proliferación de unidades de transporte "pirata" responde, en parte, a la necesidad económica de miles de desempleados, así como a intereses de políticos y líderes sindicales, quienes conciben a los trabajadores del volante como clientela electoral susceptible de participar en movilizaciones políticas o como grupos de choque.

Hasta 2017, "el parque vehicular que circulaba en territorio del Estado de México se integraba por 12,796 autobuses, 5,374 microbuses, 37,405 combis y 99,155 automóviles, así como tres líneas del STC Metro ya señaladas que penetraban en el Estado de México, además del sistema del Mexibús, Mexicable y seis centros de transferencia modal" (Secretaría de Movilidad del Estado de México, 2017).

Tras este breve antecedente histórico, el objetivo central del presente ensayo consiste en evaluar el impacto del sistema Mexibús sobre la movilidad de personas en términos de costo, tiempo, funcionalidad, seguridad, eficiencia, tráfico vehicular y calidad del servicio.

Se parte del siguiente cuestionamiento: ¿Hasta qué grado el sistema de transporte público que ofrece el Mexibús ha sido una solución viable 
a la creciente demanda de traslado masivo de los mexiquenses? La hipótesis a comprobar sería que el Mexibús ha cumplido parcialmente con los objetivos que le dieron origen en cuanto a rapidez, comodidad, economía, eficiencia, seguridad, contaminación ambiental y operación en general.

\section{LA CRISIS ECONÓMICA EN EL MUNDO GLOBALIZADO}

El capitalismo ha logrado impulsar el desarrollo de las fuerzas productivas, a través del avance constante de la ciencia y la tecnología que han estimulado el incremento de los medios de producción; sin embargo, el desarrollo del capitalismo no ha sido permanente ni constante, sino interrumpido por crisis cada vez más profundas y prolongadas.

De acuerdo con Marx, si la crisis económica se define como una ruptura o un bloqueo en la reproducción del capital social ${ }^{2}$ en su conjunto, que a su vez se traduce en una menor acumulación de capital ${ }^{3}$,"se debe al grado de confluencia y agudización de las contracciones fundamentales que ha alcanzado el capitalismo" (Guillén, 1985: 71).

La crisis económica es inherente al sistema capitalista y cualquier economía presenta una dinámica que cambia a través del tiempo, a veces a saltos cortos y otras, largos, que interrumpen la acumulación del capital; a esto se le conoce como ciclos económicos, expansiones que ocurren simultáneamente en varias actividades económicas seguidas de recesos generales, contradicciones y recuperaciones que se convierten en la fase de expansión del siguiente ciclo.

Dicha recesión estructural de carácter global tuvo su máxima expresión durante la crisis financiera de 2008-2009, iniciada en Estados Unidos y que pronto se extendió por todo el mundo, mediante la caída de las bolsas de valores del mundo, en parte debido a una sobreinversión en títulos inmobiliarios especulativos.

2 El Capital es una relación social que históricamente surge cuando los medios de producción y la fuerza de trabajo son adquiridos por el capitalista para unirlos durante el proceso productivo (Marx, 1979: 616).

3 La acumulación de capital es un proceso mediante el cual se realiza la transformación de la plusvalía en capital en escala ampliada por el que todos los factores que contribuyen a elevar la plusvalía incrementan la acumulación del capital" (Marx, 1979: 761). 
Con la profundidad de la crisis económica de los ochenta en el mundo y los dictámenes del Consenso de Washington (véase Tello, 2007: 546), el proceso de la apertura comercial y la globalización se aceleraron con la adopción de una política económica de corte neoliberal, mediante la cual el Estado se retrae y el mercado adquiere un mayor protagonismo en la economía.

La globalización económica es el resultado de una nueva división internacional del trabajo que se ha desarrollado paralelamente a la internacionalización del capital, hasta generar una reconfiguración geoeconómica, cuyas principales características son: la revolución informática, capitalismo posfordista, unificación/regionalización del mercado mundial, producción internacional integrada (cadenas de valor), titularización del crédito, aceleración de los procesos migratorios e incluso mayor interdependencia ecológica mundial (Dabat y Toledo, 1999: 13).

En México y el resto del mundo, el papel del Estado también se ha transformado sensiblemente con la aplicación de políticas de corte neoliberal, debido en parte a que el Estado de Bienestar entró en crisis fiscal, por lo que fue sometido a una serie de reformas estructurales que permitieran su adecuado funcionamiento mediante "la eliminación de empresas públicas ineficientes, privatización de empresas públicas no estratégicas, reducción del gasto público y del gasto social, además de disminuir las regulaciones de la economía de mercado, liberar el comercio exterior y el sistema financiero, así como imponer el trabajo flexible acorde a los cambios tecnológicos: todo ello en el marco de una economía abierta de Estado mínimo" (Ávila, 2006: 22).

En síntesis, con la política neoliberal y la globalización, el Estado se retrajo y el mercado se expandió en beneficio de las empresas transnacionales y del capital extranjero, quienes estimularon una mayor concentración del ingreso, así como las ganancias entre unos cuantos, a expensas del incremento de la pobreza, el desempleo, las desigualdades y una mayor pobreza entre gran parte de la población. 


\section{CRISIS URBANA Y gobernANZA POLÍtICA}

Las fallas en los servicios públicos urbanos, como el transporte, el drenaje, el suministro de agua potable, la recolección de basura o la seguridad pública, causadas por algún imprevisto meteorológico o social, después de una larga jornada de trabajo, escolar o de alguna actividad cotidiana de una gran ciudad desquician el regreso a casa, lo cual nos recuerda que vivimos en una crisis urbana latente, producto de los altos niveles de concentración y densidad demográfica, así como de una alta desigualdad económica, que hacen compartir el mismo espacio urbano a clases muy pudientes y muy pobres. Esta situación que Hirsch llama caos urbano, "se presenta en toda comunidad urbana, enormemente compleja y a veces incontrolable, puesto que abarca varios niveles de gobierno que casi siempre están al borde del caos" (1977: 17).

La crisis urbana surge cuando la organización material de la vida cotidiana de los habitantes urbanos aparece cada vez más en contracción con los deseos y aspiraciones populares y con la funcionalidad del sistema; es decir, tiene su origen en las contradicciones económicas del capitalismo. "Existe crisis urbana a partir del momento en que la organización de las grandes ciudades contradice los valores y necesidades de la mayor parte de los grupos sociales, mientras que al mismo tiempo dificulta la realización de los objetivos que asignan al sistema urbano los intereses estructurales dominantes. Es decir, se trata de una crisis que de forma simultanea dificulta el desarrollo del sistema económicosocial y socava sus fuentes de la legitimación política" (Castells, 1981: 1).

La raíz estructural de la crisis urbana surge en el momento en que la producción y distribución de los medios de consumo necesarios para la reproducción de la fuerza de trabajo, como vivienda, agua, drenaje, educación, salud, electricidad, recolección de basura y servicios de transporte, no son rentables para el capital privado; por ello se justifica la intervención del Estado para producir, distribuir y gestionar los bienes y servicios que la sociedad demanda.

En este sentido la intervención del Estado en su afán de paliar en parte los estragos de la crisis urbana, es profundamente contradictoria, primero porque la redistribución del valor que el Estado realiza de esta forma, entra en contradicción con la norma capitalista de la apropiación privada del excedente económico; segundo, al ser 
el Estado expresión de los conflictos de poder y de los conflictos sociales en su seno, los sistemas urbanos oscilarán en función de la correlación de fuerzas y se convertirán en escenario y objeto de la extensión de la lucha de clases (Castells, 1981: 321).

Con la aplicación de la política neoliberal se ha perpetrado un impacto directo sobre las principales funciones del Estado en la organización económica, política, social y territorial, al pasar de un Estado interventor a uno facilitador. Este modelo ha implicado la creciente presencia de empresas transnacionales en las ciudades subdesarrolladas, dado que con mayores inversiones crece la demanda de suelo urbano, así como obras de infraestructura que garanticen el funcionamiento del aparato productivo, equipamiento colectivo que permita la reproducción de la fuerza de trabajo, además de diversos servicios.

Esta política se ha manifestado en un mayor protagonismo de las empresas constructoras privadas, en la generación de nuevas viviendas, así como en la edificación de gasolineras, hoteles, autoservicios, hospitales y escuelas, hasta convertirse de facto en los auténticos planeadores del desarrollo urbano y regional del país, ante la ausencia o apatía del Estado neoliberal, quien sólo desempeña el papel de simple facilitador de la acumulación y valorización del capital privado.

Esta contradicción, producto de la globalización económica, ha provocado profundas modificaciones en la estructura económica y acceso a servicios básicos, además del crecimiento de la mancha urbana y funcionalidad de las grandes urbes, sobre todo subdesarrolladas, en donde ha crecido el deterioro del equipamiento urbano, hacinamiento habitacional, escasez de agua, saturación del transporte público, mayor tráfico vehicular y contaminación, así como el deterioro de las condiciones de vida de la mayoría de los habitantes urbanos, lo cual ha provocado que la gobernanza de las metrópolis estén entre las exigencias de la globalidad y las demandas de la población.

\section{CONDICIONES GENERALES DE LA PRODUCCIÓN}

El desarrollo del sistema capitalista requiere localizarse sobre una base material o territorial, lo cual implica la existencia de diversas obras de infraestructura y equipamiento urbano (capital social), para poder hacer factible los procesos de producción, distribución, circulación y 
consumo de bienes y servicios que demanda la sociedad. "El capital social tiene un doble carácter, tanto como factor de la producción, así como condición general de la producción geográficamente localizada, se constituye en una categoría histórica que permite articular una teoría unificada del desarrollo económico y la organización espacial del proceso productivo" (Garza, 2014: 14).

Como factor de la producción, el capital, junto con la fuerza de trabajo, la tecnología, la tierra y el conocimiento, constituyen los principales factores tradicionales de la producción que posibilitan la elaboración de bienes y servicios demandados, así como la valorización del capital. Sin embargo, como condición general de la producción, el capital busca resolver de antemano y garantizar la existencia de todas las obras de infraestructura y equipamiento que posibiliten la producción y circulación del capital, pero sin ser financiadas por el capital privado, sino por el público. De manera precisa, las condiciones generales de la producción "son aquellas condiciones de producción que no forman parte de los medios al interior de las empresas, puesto que el capital busca siempre solo las condiciones particulares de su valorización; las colectivas las transfiere al país entero en calidad de necesidades nacionales" (Marx, 1982: 20).

La construcción de las condiciones generales de la producción como capital constante fijo están estrechamente vinculadas con la expansión de los medios de producción privados, $\mathrm{y}$ en conjunto, transforman las ciudades en verdaderas fuerzas productivas que constituyen los nodos espaciales articuladores del desarrollo económico; implica una gran inversión en diversas obras de infraestructura, tales como abasto de agua y servicios de drenaje, sistema eléctrico, dotación de hidrocarburos, red vial y tejido urbano, transporte urbano y sistema telemático, entre otros" (Garza, 2014: 14).

Adicionalmente, se requiere la edificación de los medios de consumo colectivos que permitan la reproducción de la fuerza de trabajo mediante la construcción de vivienda pública, dotación de agua potable, electricidad, drenaje, así como equipamiento para los servicios de salud, educación, cultura, esparcimiento y transporte urbano. 


\section{CRISIS ECONÓMICA, URBANA Y FINANCIAMIENTO DE LAS CONDICIONES GENERALES DE LA PRODUCCIÓN}

La crisis económica permanente limita considerablemente la capacidad de respuesta de los gobernantes de estas metrópolis, cuyo presupuesto limitado les impide financiar costosas obras, como transporte público; esto forma parte de lo que Marx denomina "condiciones generales de la producción", pero el rápido crecimiento demográfico y la gradual demanda de servicios urbanos básicos provocan que la ciudad esté al borde del caos en el marco de una crisis urbana permanente, como lo señala Hirsch.

La gobernanza de la ZMCM, así como la de todas las metrópolis de América Latina, enfrentan grandes retos y desafíos que cada vez son más difíciles de superar, sobre todo por las progresivas dificultades para mantener un crecimiento económico sostenido, lo que genera niveles de inversión y producción necesarios, así como de ingresos y empleos que demanda la población creciente.

\section{El SISTEMA DEL TRANSPORTE PÚBlico MEXIBÚS EN EL ESTADO DE MÉXICO}

\subsection{Metodología}

Con el propósito de brindar el sustento estadístico a la investigación, además de comprobar nuestra hipótesis de trabajo, se aplicaron dos encuestas de campo, la primera orientada a recabar las opiniones y sugerencias de los usuarios frecuentes del Mexibús, que recorren diariamente los municipios de Ecatepec, Tecámac, Coacalco, Tultitlán, Cuautitlán, Nezahualcóyotl y Chimalhuacán, en relación con los temas siguientes: motivos de viaje, características laborales, nivel educativo, costos de viaje y evaluación cualitativa del servicio del Mexibús.

Conocido el aforo de usuarios frecuentes que se registra de lunes a viernes en las tres líneas del Mexibús, se procedió a determinar el tamaño de muestra para poblaciones conocidas:

$$
n=\frac{N Z^{2} p q}{(N-1) \varepsilon^{2}+Z^{2} p q}
$$




\section{Donde:}

$\mathrm{n}=$ será el tamaño de muestra buscado

$\mathrm{N}=$ corresponde al total de la población absoluta promedio que diariamente utiliza el Mexibús

$Z_{\alpha}=$ nivel de confianza estadístico

$\mathrm{p}=$ se refiere a la proporción esperada del $5 \%$, en (donde $\mathrm{q}=1-\mathrm{p}=95 \%$

$\mathrm{q}=1-\mathrm{p}$

$\varepsilon=$ error de muestreo aceptable.

De esta manera, la cantidad del primer tipo de encuesta aplicada entre los usuarios para cada una de las líneas del Mexibús y las fechas correspondientes queda de la forma siguiente:

Línea 1: Cd. Azteca-Ojo de Agua; del 4 al 8 de julio de 2016:

\section{1,437 encuestas}

Línea 2: Coacalco-Las Américas; del 11 al 15 de julio de 2016:

\section{1,423 encuestas}

Línea 3: Pantitlán-Chimalhuacán; del 18 al 22 de julio de 2016:

\section{1,423 encuestas}

\section{Total: 4,283 encuestas}

Todas las encuestas que sustentan esta investigación fueron aplicadas del 4 al 22 de julio de 2016, de lunes a viernes, de 09:00 a 15:00 horas, horario durante el cual se registra un considerable nivel de ocupación del transporte; el levantamiento se realizó en las estaciones terminales, los pasillos y a bordo de las unidades. La segunda encuesta se aplicó simultáneamente entre los diversos comerciantes de bienes y servicios que poseen o alquilan un local establecido en las vialidades por donde circulan las unidades del Mexibús, con el objeto de recabar sus opiniones y sugerencias sobre el transporte articulado. El objetivo principal de la aplicación de esta segunda encuesta fue conocer el giro del negocio, antigüedad en la zona, tipo de local, precios e incrementos del alquiler del local comercial, impacto del Mexibús en el nivel de ventas, así como quejas y sugerencias al respecto.

Bajo el criterio de observación y conteo de negocios existentes, se determinó aplicar 10 encuestas para cada estación, cinco en cada acera, cuyo resultado fue el siguiente: 
Línea 1: Cd. Azteca-Ojo de Agua; -> 24 estaciones -> 240 encuestas Línea 2: Coacalco-Las Américas; $->43$ estaciones $->430$ encuestas Línea 3: Pantitlán-Chimalhuacán; -> 29 estaciones $->290$ encuestas Total: 960 encuestas

En general, la aplicación de más de cinco mil encuestas en total entre la 1 y la 2 arrojaron como resultados diversas fallas e inconsistencias, así como aciertos y sugerencias del Mexibús en la opinión de los usuarios. Con las encuestas, así como con el uso de la técnica costobeneficio, se detectaron como conclusiones parciales diversos aspectos positivos del transporte público, como la seguridad, rapidez y el comercio, así como aspectos negativos, entre los que destacan el mayor tráfico vehicular, el tiempo de espera, el doble cobro en la conectividad y la falta de cortesías para usuarios de la tercera edad, entre otros.

\subsection{Líneas del Mexibús}

\section{Línea 1: Ciudad Azteca-Ojo de Agua}

La importancia y la planeación de esta línea no sólo obedece a haber sido la primera de su tipo que fue puesta en operación en todo el Estado de México del sistema de transporte masivo Mexibús en octubre de 2010, sino a que se construyó en el municipio de Ecatepec, el de mayor población en la entidad, lo que permitió conectarse con Tecámac, región vecina que ha resentido los estragos del capital inmobiliario desde hace más de 30 años. Esta línea tiene una extensión de $17.5 \mathrm{~km}$, integrada por 24 estaciones que corren por Ecatepec y Tecámac (Mapa 1).

Durante el transcurso de esas tres décadas, la población de Ecatepec ha crecido más de $37.7 \%$; pasó de 1,218,135, en 1990, hasta 1,677,678 habitantes, en $2015,10.4 \%$ de la población total de la entidad en ese último año; mientras que Tecámac vio crecer su población $26.2 \%$; de 123,218 , en 1990, pasó a 446,008 habitantes, en 2015, lo cual representó $2.8 \%$ del total del Estado de México.

Ante el crecimiento desbordante, tanto demográfico como urbanístico, en Ecatepec y Tecámac en las recientes tres décadas, se hizo necesaria la introducción del autobús de tránsito rápido (BRT por sus siglas en inglés) en el Estado de México, que ya opera en diversas ciudades del mundo, incluyendo el sistema Metrobús de la Ciudad de México introducido en 2005. Resultaba hasta cierto punto lógico que la primera línea diseñada del Mexibús beneficiaría a Ecatepec por ser el muni- 
cipio más poblado y con mayor demanda de servicios de transporte en el Estado de México, además de Tecámac, que ha registrado elevadas tasas demográficas y mancha urbana desbordante.

\section{MAPA 1}

Línea 1: M. Ciudad AzTeCA-OJo de Agua

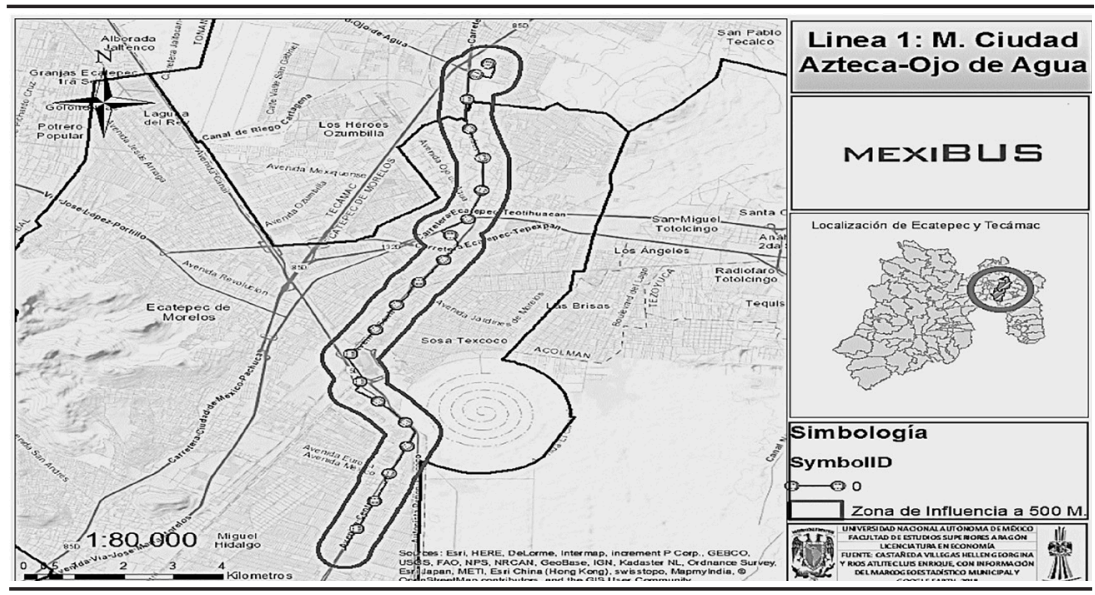

Fuente: Castañeda Villegas Hellen Georgina y Ríos Atlitec Luis Enrique, con base en el Margo Geostadístico Municipal y Google Earth, 2018.

En el Cuadro 1 se muestra la opinión de los usuarios sobre diversas condiciones y características del servicio del Mexibús; se observan diversas respuestas contrastantes, por ejemplo, $92.6 \%$ aprueba el estado de las unidades; $92.2 \%$ aprueba las condiciones de las instalaciones, y a $90.4 \%$ les parece adecuado el horario de servicio; situación que contrasta con $83.5 \%$ de aprobación de los pasajeros de la atención de los conductores, $69.2 \%$, las formas de pago, y, sobre todo, $52.3 \%$, la cantidad de pasajeros permitida en cada unidad. Estos tres últimos aspectos que registran altos niveles de desaprobación representan las quejas más recurrentes de los usuarios.

A pesar de los múltiples reclamos sobre la aglomeración dentro de las unidades, formas de pago y atención de los conductores, los usuarios están $90.5 \%$ a favor de que se sigan construyendo más líneas del Mexibús, contra la opinión de $7.7 \%$ que no está de acuerdo. 
CUADRO 1

GRADO DE APROBACIÓN Y DESAPROBACIÓN DEL SERVICIO SEGÚN LA OPINIÓN DE USUARIOS

\begin{tabular}{ccc}
\hline Concepto & Aprueba & Desaprueba \\
\hline Estado de las unidades & 92.6 & 7.1 \\
\hline Condiciones de las instalaciones & 92.2 & 7.6 \\
Horarios de servicio & 90.4 & 9.2 \\
\hline Atención de conductores & 83.5 & 16.3 \\
\hline Formas de pago & 69.2 & 30.4 \\
\hline Cantidades de pasajeros permitidos & 52.3 & 47.3 \\
\hline
\end{tabular}

Fuente: elaboración propia con base en las encuestas aplicadas.

Nota: la suma horizontal puede no corresponder a $100 \%$ por aquellos usuarios que no contestaron.

\section{Línea 2: Coacalco-Las Américas}

De las tres líneas del sistema de transporte del Mexibús analizadas en el presente estudio, esta ruta es la más larga: 21.3 kilómetros y 43 estaciones instaladas a lo largo de cuatro municipios: Ecatepec, Coacalco, Tultitlán y Cuautitlán, además de haber sido inaugurada recientemente, enero de 2015 (Mapa 2). Desde su puesta en operación, esta línea fue objeto de severas críticas y cuestionamientos por parte de los usuarios en particular, sobre todo porque fue abierta al público cuando aún tenía algunas estaciones sin concluir en cuanto albañilería, pintura, yeso, rampas de acceso, máquinas, torniquetes, seguridad e incluso escasez de unidades de transporte; obras inconclusas que llegaron a provocar diversos accidentes entre los usuarios. 
MAPA 2

Línea 2: Plaza las AmÉRICAS-LA Quebrada

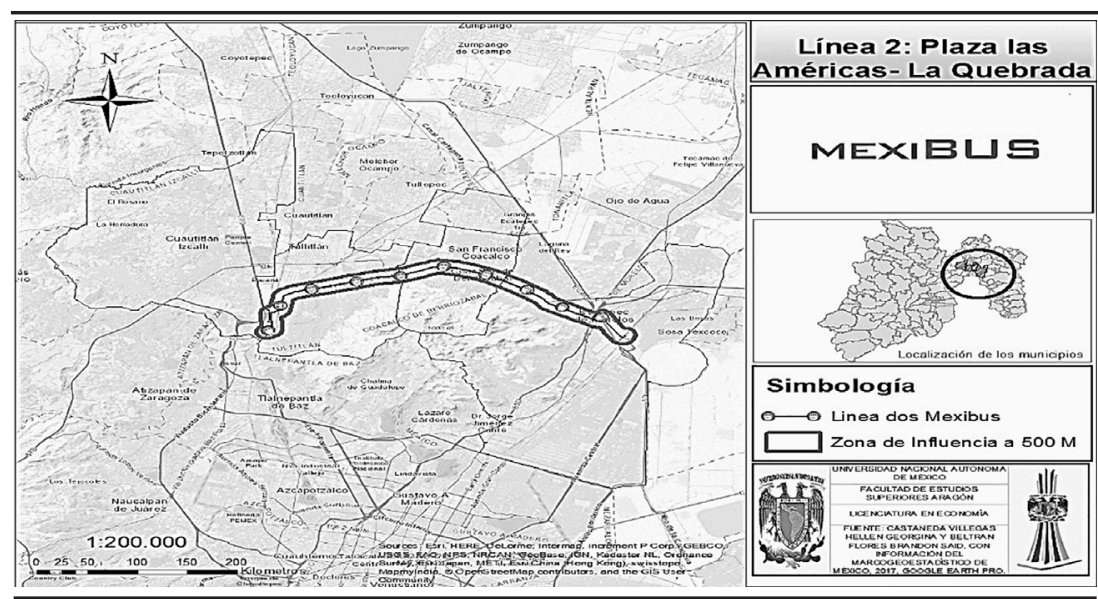

Fuente: Castañeda Villegas Hellen Georgina y Ríos Atlitec Luis Enrique, con base en el Margo Geostadístico Municipal y Google Earth, 2018.

Durante el lapso mencionado, la población de Ecatepec creció 7.8 veces; pasó de 216,408 habitantes, en 1970, a 1,677,678, en 2015; seguido por Coacalco, cuya población creció 21.5 veces; pasó de 13,197 a 284,462 habitantes; mientras que Tultitlán vio crecer 10 veces su población; de 52,317 aumentó a 520,557; finalmente, Cuautitlán creció 3.6 veces, de 41,156 habitantes pasó a 149,550 durante el periodo 1970-2015. Cabe señalar que los cuatro municipios estudiados contaban conjuntamente 2,632,247 habitantes, es decir, $16.3 \%$ de la población total del Estado de México hasta 2015.

Debido al explosivo crecimiento urbano y demográfico que ha registrado la zona, fue necesaria la construcción y arranque de la línea 2 del Mexibús en enero de 2015, lo que, aunado a la puesta en marcha del tren suburbano en mayo de 2008, que sale de la antigua estación ferroviaria de Buenavista y llega al municipio de Cuautitlán, Estado de México, mejoró notablemente el transporte público de los miles de pasajeros de la zona. 
CUADRO 2

GRADO DE APROBACIÓN Y DESAPROBACIÓN DEL SERVICIO SEGÚN LA OPINIÓN DE USUARIOS

\begin{tabular}{ccc}
\hline Concepto & Aprueba & Desaprueba \\
\hline Estado de las unidades & 95.8 & 4.1 \\
\hline Condiciones de las instalaciones & 95.1 & 4.7 \\
Horarios de servicio & 90.9 & 9.0 \\
\hline Atención de conductores & 90.7 & 9.2 \\
\hline Formas de pago & 68.9 & 31.0 \\
\hline Cantidades de pasajeros permitidos & 68.7 & 31.1 \\
\hline
\end{tabular}

Fuente: elaboración propia con base en las encuestas aplicadas.

Nota: la suma horizontal puede no corresponder a $100 \%$ por aquellos usuarios que no contestaron.

Con el propósito de profundizar en la opinión de los usuarios respecto a la calidad del servicio de transporte que brinda el Mexibús, en el Cuadro 2 se muestran diversas variables que permitieron conocer el grado de aprobación y desaprobación del servicio. El estado de las unidades obtuvo la mayor aprobación de los usuarios con 95.8\%; los horarios de servicio, 95.1\%; la atención del conductor, 90.9\%, y finalmente las condiciones de las instalaciones, $90.7 \%$. Contrariamente, los servicios peor calificados fueron tanto la cantidad de pasajeros permitidos, como las formas de pago, con $68.9 \%$ y $68.7 \%$, respectivamente.

Obviamente los aspectos peor calificados deberían ser objeto de mayor atención por parte de las autoridades que administran el Mexibús, con la incorporación de más unidades de transporte para evitar las aglomeraciones en cada autobús, así como implementar un sistema de recarga de tarjetas o modernizar las máquinas actuales similares a las existentes en el mercado de la tecnología.

De las sugerencias hechas por los usuarios y locatarios, destaca que $29 \%$ de ellos solicita más y mejores señalamientos de tránsito y peatonales; seguidos por $13 \%$ que denuncia el mal funcionamiento de las máquinas expendedoras de tarjetas; mientras que $11 \%$ de otro sector de la población pide excluir los demás transportes públicos que circulan paralelamente al Mexibús, como sucede en la línea 1, ya que entorpecen el tráfico vehicular, generan mayor contaminación y provocan 
accidentes; estas sugerencias, aunadas a las de $10 \%$ que pide mayor regularidad en tiempos y otras diversas sugerencias, sumaron $73 \%$ del total de sugerencias. Con menor frecuencia, los usuarios recomendaron una mayor vigilancia, operadores mejor preparados, incluir más unidades, mejorar la limpieza y el mantenimiento; incluso 5\% solicitó eliminar el Mexibús.

\section{Línea 3: Pantitlán-Chimalhuacán}

En el Mapa 3 se muestra la línea más corta, con $14.5 \mathrm{~km}$ de extensión y 29 estaciones instaladas a lo largo de los municipios de Nezahualcóyotl y Chimalhuacán, cuya terminal se encuentra en el paradero de Pantitlán, delegación Venustiano Carranza. Es la única línea del Mexibús que ingresa a la Ciudad de México. De las 29 estaciones que comprende, 16 se localizan en Nezahualcóyotl, 12 en Chimalhuacán y una en la Ciudad de México.

\section{MAPA 3}

\section{LÍNEA 3: M. PANTITLÁN-CHIMALHUACÁN}

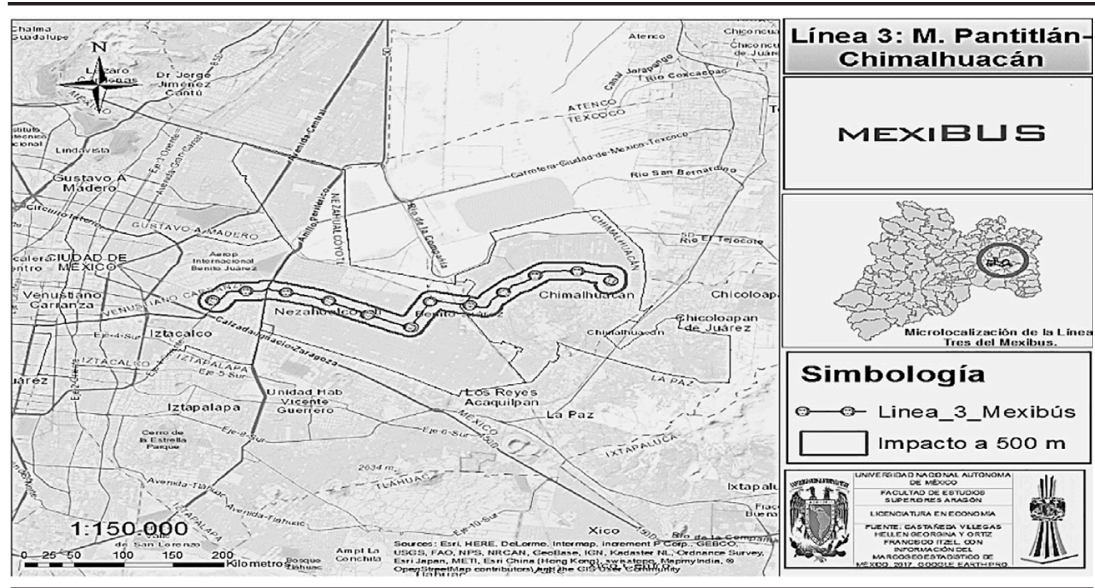

Fuente: Castañeda Villegas Hellen Georgina y Ríos Atlitec Luis Enrique, con base en el Margo Geostadístico Municipal y Google Earth, 2018. 
La expansión urbana de estas demarcaciones se suscitó en el periodo 1960-1970, como resultado del crecimiento de la Ciudad de México hacia la zona oriente, en donde había amplios terrenos cercanos, planos y baratos, producto de la desecación paulatina del Lago de Texcoco, e impulsado adicionalmente por los crecientes flujos migratorios campociudad entre 1940 y 1980.

Durante el periodo 1970-2015, la población de Chimalhuacán creció más de 34 veces; pasó de 19,946 habitantes, en 1970, a 679,811, en 2015; mientras que Nezahualcóyotl lo hizo en 1.8 veces; de 580,436, en 1970, pasó A 1'039,867 habitantes, en 2015; contrariamente, la delegación Venustiano Carranza registró una reducción de su población de 0.59 veces; pasó de 721,529 habitantes, en 1970, a 423,263, en 2015, periodo durante el cual arrojó tasas demográficas de crecimiento negativas.

Considerando las altas tasas de crecimiento demográfico mostradas por ambos municipios, así como la rápida urbanización de sus territorios y la creciente demanda de servicios de transporte, fue necesaria la construcción y puesta en marcha de la línea 3 del Mexibús, la cual fue inaugurada en abril de 2013, misma que inicia su recorrido en la estación del metro Pantitlán, en la Venustiano Carranza, atraviesa Nezahualcóyotl y llega al municipio de Chimalhuacán.

\section{CUADRO 3}

GRADO DE APROBACIÓN Y DESAPROBACIÓN DEL SERVICIO SEGÚN LA OPINIÓN DE USUARIOS

\begin{tabular}{ccc}
\hline Concepto & Aprueba & Desaprueba \\
\hline Estado de las unidades & 93.2 & 6.8 \\
\hline Condiciones de las instalaciones & 92.0 & 7.8 \\
\hline Horarios de servicio & 89.9 & 10.1 \\
\hline Atención de conductores & 76.1 & 23.6 \\
\hline Formas de pago & 69.4 & 30.5 \\
\hline Cantidades de pasajeros permitidos & 36.0 & 63.8 \\
\hline
\end{tabular}

Fuente: elaboración propia con base en las encuestas aplicadas.

Nota: la suma horizontal puede no corresponder a $100 \%$ por aquellos usuarios que no contestaron. 
Conforme a los resultados mostrados en el Cuadro 3, referente a la opinión de los usuarios de la línea 3 , la variable mejor calificada fue la correspondiente a los horarios de servicio, con 93.2\%, seguido por la atención de los conductores, 92\%; mientras que el estado de las unidades fueron aprobados con $89.9 \%$, las condiciones de las instalaciones, $76.1 \%$, y las formas de pago aprobadas con $69.4 \%$, contra $36 \%$ que sólo aprueba la cantidad de pasajeros permitidos.

Resulta evidente que tanto las formas de pago como la cantidad de pasajeros permitidos, al ser los servicios peor calificados, deberían ser objeto de atención y modificación por parte de las autoridades administradoras, con el claro propósito de mejorar y hacer más eficiente el servicio. Lo anterior se justifica con la opinión de los usuarios; 94.3\% respondió que sí debieron seguirse construyendo más líneas del Mexibús, contra 5.5\% que se opone a ello.

Entre las principales quejas y sugerencias que los usuarios hacen destaca $20 \%$ que solicitó más unidades, seguido por $13 \%$ que pide un mejor funcionamiento de las máquinas de cobro, y otro porcentaje similar solicitó más y mejores señalamientos de tránsito y peatonales, incluso $11 \%$ requiere mayor vigilancia en las estaciones, así como mejor consistencia en los tiempos de las corridas de las unidades; todas estas sugerencias sumaron $68 \%$ del total. El resto de éstas varió desde tener operadores más precavidos para evitar accidentes, hasta atender las necesidades de los negocios, cambiar de ruta al Mexibús y brindar tarifas más bajas; todas sumaron $29 \%$, complementada por $3 \%$ que sugirió quitar el servicio del Mexibús.

Mediante el anális de cada línea de Mexibús, podemos adelantar una conclusión sobre los giros mercantiles que las caracterizan, por su número y naturaleza. Bajo esta perspectiva, la línea 1 destacó porque en el recorrido predominaron: las tiendas de abarrotes, refaccionarias, tiendas de pinturas y de conveniencia, así como gasolineras y gaseras.

En la línea 2 del Mexibús predominan grandes centros comerciales, agencias automotrices, gasolineras, tiendas de conveniencia, además de las sucursales bancarias; todos éstos son establecimientos que denotan un nivel socioeconómico medio y medio-alto de los habitantes.

En contraste, en la línea 3 del Mexibús, donde prevalecen tiendas de abarrotes, refaccionarias, tiendas de pinturas, farmacias y sobre todo casas de préstamo y ahorro, el nivel socioeconómico que subyace es el 
bajo, que en promedio registran las familias de las colonias populares por donde circula el Mexibús.

\subsection{Costos totales e inversión}

En lo general, podemos ubicar al Mexibús como parte de las Condiciones Generales de la Producción, desarrolladas y definidas originalmente por Marx como capital constante fijo vinculado estrechamente con la expansión de los medios de producción privados, quienes en conjunto transforman las ciudades en verdaderas fuerzas productivas, que constituyen los nodos espaciales articuladores del desarrollo económico.

Conforme a la metodología propuesta por Garza (2014), el transporte urbano, como el Mexibús, tiene un carácter ambivalente, dado que forma parte tanto de los medios de trabajo socializados requeridos por el aparato productivo, a través de las condiciones generales de la circulación, como de los medios de consumo colectivo necesarios para la reproducción de la fuerza de trabajo; en la medida en que su razón de ser obedece al transporte de pasajeros desde su lugar de residencia hacia el trabajo o la escuela, para realizar compras, llevar a cabo diversos trámites administrativos, entre otros.

Es muy complejo analizar la operación del sistema de transporte urbano en la ZMCM, tanto por el número de horas/viaje/día de los millones de pasajeros que se transportan cotidianamente, además del tránsito vehicular y la contaminación ambiental generada por millones de automotores, así como por la dificultad político-administrativa que significa separar el servicio de transporte que se presta en la zona conurbada del Estado de México y en la CDMX, que integran la ZMCM.

El grado de complejidad aumenta si pretendemos comparar los costos totales que ha implicado la puesta en marcha del sistema de Autobuses de Tránsito Rápido, conocido como Metrobús en la Ciudad de México y Mexibús en el Estado de México; máxime cuando el primero entró en operación desde junio de 2005, y ya contaba con siete líneas hasta marzo de 2018; mientras que el Mexibús, que presta el servicio en los municipios más poblados del Estado de México, apenas ha logrado construir cuatro líneas de transporte articulado desde octubre de 2010 hasta 2019.

Evidentemente existe una situación de contrastes en ambas entidades en materia de transporte tipo BRT; por un lado, el Metrobús opera 
en la Ciudad de México siete líneas para atender a 8.9 millones de habitantes, contra cuatro líneas del Mexibús en la zona conurbada del Estado de México para dar servicio a más de 16.1 millones de mexiquenses.

Independientemente de los contrastes y diferencias señaladas entre ambos sistemas BRT, para fines del presente ensayo, nos concentramos en el análisis de costos del Mexibús. En la línea 1, que corre de Ciudad Azteca a Ojo de Agua, el costo de la inversión inicial ascendió a 1,490.6 millones de pesos; la construcción del carril confinado para la circulación del autobús requirió 33.3\% del costo total, seguido por el de las unidades BRT, con $19.2 \%$, el de las obras viales y complementarias, con $13.4 \%$, y el del centro de control, que implicó $0.4 \%$ de la inversión total.

Del costo total de la línea 2, que ascendió a 1,668.2 millones de pesos, destaca la inversión para la edificación del carril confinado, con $28.4 \%$, seguido por el costo de los equipos BRT, $25.7 \%$, mientras que la construcción de las obras viales y complementarias absorbió $11.35 \%$, y la de las estaciones, $10.58 \%$ de la inversión total, contra $0.4 \%$ por la edificación del centro de control (Cuadro 4).

Para construir la línea 3 del Mexibús se requirió hacer una inversión total de 1,484 millones de pesos; de los cuales $28.2 \%$ se destinó a la construcción del carril confinado, seguido por el costo de los equipos BRT, que implicaron $16.7 \%$, mientras que la edificación de las obras viales y complementarias absorbieron $10.55 \%$, y la de las estaciones, $8.36 \%$ de la inversión total; contra $0.4 \%$ del costo de construir el centro de control (Cuadro 4).

Si efectuamos el análisis comparativo de las tres líneas del Mexibús, apoyándonos en la Tabla 4, destacan algunos aspectos relevantes; en primer lugar, la línea 2 es la más extensa del sistema con $21.3 \mathrm{~km}$; cuenta con el mayor número de estaciones con 43; tuvo un costo mayor, 1,668.2 miles de pesos, pero ocupó el segundo lugar con 95,586 pasajeros transportados, y registró 15,924 pesos en el costo por pasajero. 
CUADRO 4

COSTO TOTAL Y COSTO POR PASAJERO TRANSPORTADO SEGÚN LÍNEA DEL MEXIBÚS

\begin{tabular}{cccccc} 
Concepto & $\mathrm{Km}$ & $\begin{array}{c}\mathrm{N}^{\circ} \text { de } \\
\text { Estaciones }\end{array}$ & Costo total & $\mathrm{N}^{\circ}$ de pasajeros & $\begin{array}{c}\text { Costo por } \\
\text { pasajero }\end{array}$ \\
\hline Línea 1 & 17.5 & 24 & $\$ 1,490,603.44$ & 121,144 & $\$ 11,226$ \\
\hline Línea 2 & 21.3 & 43 & $\$ 1,668,261.66$ & 95,586 & $\$ 15,924$ \\
\hline Línea 3 & 14.5 & 29 & $\$ 1,471,261.62$ & 65,574 & $\$ 23,474$ \\
\hline Total & 53.3 & 96 & $\$ 4^{\prime} 421,365.01$ & 282,304 & $\$ 15,662$ \\
\hline
\end{tabular}

Fuente: elaboración propia con base en Transconsult ( $a, b, c)$. "Estudio de evaluación, diseño funcional y operacional del BRT del corredor de transporte público".

Mientras que la línea 1 del Mexibús registró menos estaciones; fue la segunda en extensión total, con $17.5 \mathrm{~km}$, y un costo total de $1,490.6$ miles de pesos; sin embargo, se colocó en primer lugar en el número de pasajeros transportados, con 121,144, y la de menor costo per cápita al registrar 11,226 pesos por pasajeros.

Finalmente, la línea 3 del Mexibús resultó ser la que menor número de pasajeros transportó, 65,574; cuenta con 29 estaciones existentes a lo largo de más de $14.5 \mathrm{~km}$, con un costo por pasajero de 23,474 pesos.

\subsection{Comparativo entre la situación sin proyecto contra una situación con proyecto}

Para garantizar el financiamiento de las tres líneas, los proyectos originales señalaban la necesidad de hacer participar a los permisionarios y transportistas de camiones, combis y microbuses en calidad de socios que operaran las unidades BRT, así como otra empresa privada que construyera las estaciones, comprara los torniquetes y las máquinas expendedoras de tarjetas y de recarga; todo ello con el fin de incorporarlos productivamente y reducir la posibilidad del conflicto social. De esta manera, se sugería que $59.9 \%$ de la inversión total fuera aportado con los recursos provenientes del Fonadin y $13.4 \%$ de aportaciones estatales.

Del Cuadro 5 se desprende un elemento muy importante que no fue considerado entre los beneficios del proyecto original y que va aumentando de valor con la maduración del proyecto: la seguridad e inte- 
gridad física de los usuarios. Conforme a los resultados de la encuesta, los usuarios se sienten más seguros de viajar en el Mexibús, a diferencia del transporte tradicional, tales como micros, combis y camiones, en donde están expuestos a mayor número de asaltos y accidentes en trayecto.

Otro de los aspectos que debieron haber sido considerados entre los beneficios del proyecto es la actividad comercial, la cual se incrementó con la puesta en marcha del Mexibús; sin embargo, al aplicar las encuestas, muchos locatarios se quejaron de que la circulación del Mexibús redujo un carril de la vialidad que antes era utilizado como estacionamiento por parte de los clientes, zona de reparación de automóviles, o bien, era invadido para exhibir algunos de los productos ofrecidos, como muebles, pinturas y ropa en general.

La introducción y operación del sistema de máquinas para la compra y recarga de tarjetas, que originalmente se contaban como parte de los beneficios del sistema de cobro Mexibús, con las frecuentes fallas que han mostrado las mismas y que han sido objeto de quejas frecuentes por parte de los usuarios, debieran ser consideradas como un lastre muy costoso que debieran modernizarse con el afán de eficientar el servicio del sistema (Cuadro 5).

El tiempo de espera de los pasajeros en el andén, que originalmente también estaba considerado como uno de los principales beneficios del proyecto, no ha sido la constante en el servicio de transporte Mexibús, al reportarse fuertes retrasos en la línea 3 que oscilan entre 20 y 30 minutos de espera, de 10 a 20 minutos en la línea 2, y de 5 a 10 minutos en la línea 1 del transporte articulado. Lo anterior no sólo obedece a la menor flotilla de camiones articulados de la línea 3, sino al tránsito vehicular e invasión de carriles que enfrentan las unidades del Mexibús al circular sobre las principales vialidades. 


\section{CUADRO 5}

COMPARATIVO ENTRE LA SITUACIÓN SIN PROYECTO CONTRA UNA SITUACIÓN CON PROYECTO SEGÚN RESULTADOS DE LA ENCUESTA

\begin{tabular}{ccc}
\hline Situación sin proyecto & Variable & Situación con proyecto \\
Menor seguridad & Seguridad & Mayor seguridad \\
Menor comercio & Comercio & Mayor comercio \\
Frecuentes & Paradas intermedias & Exclusivas \\
n/s/a & Operación de máquinas & Escasas y lentas \\
Menor tiempo de espera & Tiempo de espera & Mayor tiempo de espera \\
Sin cambios & Conectividad & Sin cambios \\
Mayor saturación & Saturación por unidad & Cortesías \\
Menor tránsito & Fluidez vehicular & Mayor saturación
\end{tabular}

Fuente: Fuente: elaboración propia con base en las encuestas aplicadas del 4 al 22 de julio de 2016.

En materia de conectividad, aún es prematuro hablar de algunos beneficios sustanciales, sobre todo si consideramos el amplio territorio que recorren las unidades a través de las rutas señaladas y la imperiosa necesidad de crear una verdadera red de transporte urbano Mexibús en el futuro, que no sólo tenga conectividad con las terminales del metro Ciudad Azteca (Mexibús línea 1), Pantitlán (Mexibús línea 3) y el tren suburbano (Mexibús línea 2), sino que también promueva la conectividad intermunicipal a bajo costo en tarifas, eliminando el doble pago de abordaje cuando el pasajero busca correspondencias entre la línea 1 y la línea 2 (Cuadro 5).

Otro de los factores omitidos en el proyecto original y que hoy representa una de las principales quejas de los usuarios es el de la saturación por unidad de transporte en horas pico, a tal grado que los usuarios indistintamente encuentran saturadas las unidades del Mexibús y del transporte tradicional de camión, micro y combi, situación que podría 
convertirse en mayores beneficios si el proyecto Mexibús hubiera contemplado la introducción de unidades de transporte adicionales.

En lo referente a la fluidez vehicular, el sistema Mexibús en general no se ha visto beneficiado mediante el uso exclusivo de un carril confinado si consideramos que en horas pico la invasión de carriles del Mexibús es una práctica común y motiva el retraso del servicio. Ello obedece no sólo a que el Mexibús presta servicio de transporte en los municipios más poblados de la periferia de la ZMCM, sino también a la falta de una solución integral que hubiera contemplado aspectos adicionales de la problemática que atraviesa cada municipio, y el del transporte es sólo una manifestación de un problema aún más complejo, como el de la concentración económica y demográfica en la ZMCM.

Finalmente, otro aspecto omitido por el proyecto original y que los usuarios asumen como un costo personal, sobre todo las personas de la tercera edad, es el de la ausencia de cortesías o la extensión de pago, ello obedece al costo de recuperación estimado por el proyecto original, con un sesgo mayormente privado en vez de social, tal como ocurre con el sistema integral de transporte en la Ciudad de México.

\section{CONCLUSIONES}

Para poder evaluar integralmente el servicio de transporte urbano Mexibús, en las tres líneas estudiadas, se aplicó la técnica costobeneficio, mediante la cual se obtuvieron resultados muy interesantes a través de la confrontación entre los estudios técnico-económicos de evaluación originalmente elaborados por la empresa privada Transconsult para las tres líneas y los resultados obtenidos mediante la aplicación de nuestras encuestas.

Para ello tomamos de base la información que la consultora elaboró para determinar la inversión social que requería cada una de las tres líneas del Mexibús para impulsar el proyecto; dicha estimación incluyó desde costos de terrenos, hasta carril confinado, obras viales y complementarias, terminales, estaciones, talleres y encierros, centro de control, equipo de cobro y boletaje, además de la adquisición de vehículos articulados.

Al comparar el costo o la inversión total para impulsar el proyecto de cada línea, con el número de pasajeros transportados por cada una de ellas, se obtuvo el costo aproximado por pasajero. Los resultados indi- 
caron que la línea 1 , con un costo total real de 1.5 mil millones de pesos y un traslado de más de 121 mil pasajeros diarios, produjo el costo por pasajero más económico de 11,226 pesos, seguidos por la línea 2, cuya inversión total real implicó más de 1.6 mil millones de pesos, cifra que, comparada con los más de 95 mil pasajeros, implicó un costo promedio por usuario de 15,924 pesos; finalmente, si bien la línea 3 del Mexibús resultó ser la menos económica, al implicar una inversión real total de más de 1.4 mil millones de pesos, lo que, comparado con el transporte de más de 65 mil pasajeros diarios, arrojó un costo mayor de 23,474 pesos por pasajero.

$\mathrm{Al}$ efectuar un análisis comparativo entre una situación sin proyecto y una con proyecto, evidentemente gana ésta última porque genera mayores beneficios sociales y económicos, entre los cuales destacan: la menor contaminación del ambiente, mayor velocidad promedio, menores costos de operación anual, menor tiempo de traslado de usuarios y un menor índice de accidentes mortales, así como una menor congestión vehicular, al dejar de circular combis, micros y camiones por donde circula el Mexibús, aunque sólo se aplica en la línea 1.

Al comparar el estudio de evaluación de la consultora con los resultados obtenidos mediante la aplicación de nuestras encuestas, detectamos algunas externalidades ${ }^{4}$ que no fueron contempladas por el proyecto original, tal es el caso de la seguridad, evaluada positivamente por los usuarios que viajan en el Mexibús, y el comercio, que incrementó al exterior de las estaciones del Mexibús.

Sin embargo, otros aspectos que no fueron bien calificados por los usuarios, considerados implícitamente como costos sociales, fueron, entre otros, las paradas intermedias fueron suprimida; con el transporte tradicional eran en cada esquina, pero ahora exclusivamente se hacen en cada estación del Mexibús; el tiempo de espera para abordar algún transporte era menor anteriormente y ahora es mayor con el Mexibús, sobre todo en hora pico; el tránsito era menor en una situación sin proyecto, puesto que ahora con proyecto implica mayor tráfico, debido en parte, al confinamiento del carril exclusivo del Mexibús, que redujo

4 Las externalidades son efectos externos que resultan del intercambio de recursos, bajo situaciones que no son de mercado, tratándose generalmente de intercambios involuntarios (Hirsch, 1977). 
el número de carriles disponibles para la circulación del resto de los vehículos; de igual forma, los usuarios de la tercera edad se quejan de que no hay cortesías como en los transportes colectivos de la Ciudad de México; la conectividad y la saturación del transporte se mantuvieron sin cambios, con y sin proyecto, en parte por el tráfico vehicular, invasión del carril exclusivo o por la falta de más unidades de transporte articulado, sobre todo en la línea 3. Finalmente, otro elemento concebido como fuente de modernidad y beneficios en el proyecto original fue el sistema de cobro mediante máquinas; sin embargo, por las constantes fallas de origen, este sistema ha sido objeto de quejas frecuentes por parte de los usuarios.

Indudablemente muchos han sido los costos y beneficios generados por el sistema de transporte Mexibús; sin embargo, por el reto de atender a siete municipios, cuyos habitantes representan casi $30 \%$ de la población total del Estado de México que siguen en crecimiento, queda la impresión de que este proyecto llegó tardíamente, puesto que está siendo rebasado por la realidad ciudadana que sigue demandando un transporte urbano, económico, seguro, eficiente y rápido; aunque también confiamos en que el Mexibús aún pueda generar mayores beneficios que costos sociales en el futuro; no obstante, el tiempo y la paciencia de los usuarios se agota.

\section{Bibliografía}

Ávila, José Luis (2006), "La era Neoliberal”, en Enrique Semo (coord.), Historia económica de México, núm. 6, Era, México.

Castells, Manuel (1981), Crisis urbana y cambio social, Siglo XXI, España.

Conapo (Consejo Nacional de la Población) (1998), Escenarios demográficos y urbanos de la ZMCM, 1990-2010, México.

Dabat, A. y A. Toledo (1999), Internacionalización y crisis en México, CRIM / UNAM, México.

De Mauleón, Héctor (2018), La Ciudad oculta 2, Planeta, México.

Garza, Gustavo (1990), "Metropolización en México", Revista Ciudades, núm. 6, abril-junio, México.

Garza, Gustavo (2014), Valor de los medios de producción socializados en la Ciudad de México, El Colegio de México, México.

Gobierno del Distrito Federal (2011), Anuario de Transporte y Vialidad de la Ciudad de México, México. 
Guillén Romo, Héctor (1985), Orígenes de la crisis en México 1948-1982, Era, México.

Hirsch, Werner (1997), Análisis de Economía Urbana, Instituto de Estudios de Administración Local, España.

INEGI (Instituto Nacional de Estadística y Geografía) (2018), Encuesta Origen-Destino 2017, México.

Linares Zarco, Jaime (2009), La imagen urbana de México en el siglo XXI, UNAM / Miguel A. Porrúa / H. Cámara de Diputados, México.

Marx, Karl (1979), El Capital, Siglo XXI, México.

Marx, Karl (1982), Elementos fundamentales para la crítica de la economía política (Gundrisse), Tomo II, Siglo XXI, México.

Secretaría de Movilidad del Estado de México (2017), Información del Parque Vehicular Transporte Público, México.

Tello, Carlos (2007), "Estado y desarrollo económico: México 1920-2006", UNAM, México.

Transconsult (2011), Estudio de evaluación, diseño funcional y operacional del BRT del corredor de transporte público, Dirección General de Infraestructura para el Transporte de Alta Capacidad, Secretaría de Comunicaciones, Gobierno del Estado de México, México.

Transconsult (s/fa), Estudio de evaluación, diseño funcional y operacional del BRT del corredor de transporte público Cd. Azteca-Ojo de Agua, México.

Transconsult (s/fb) Estudio de evaluación, diseño funcional y operacional del BRT del corredor de transporte público Coacalco-Plaza las Américas, México.

Transconsult. (s/fc) Corredor de Transporte Público Masivo NezahualcóyotlPantitlán-Chimalhuacán, México.

Unikel Luis, Crescencio Ruíz y Gustavo Garza (1974), El desarrollo urbano de México: diagnóstico e implicaciones futuras, El Colegio de México, México. 